\title{
Investigation on Layered Elliptical Vibration- Assisted Cutting of Micro Groove
}

Chen Zhang ( $\square$ meeczhang@nuaa.edu.cn )

Nanjing university of aeronautics and astronautics

\section{Research Article}

Keywords: Micro groove, Elliptical vibration-assisted cutting, Layered tool path, Simulation, Machining stress

Posted Date: February 18th, 2022

DOI: https://doi.org/10.21203/rs.3.rs-1352467/v1

License: (9) This work is licensed under a Creative Commons Attribution 4.0 International License. Read Full License 


\title{
Title page
}

\section{Investigation on Layered Elliptical Vibration-Assisted Cutting of Micro Groove}

\author{
Chen Zhang* \\ College of Mechanical and Electrical Engineering, Nanjing University of Aeronautics \\ and Astronautics, China, 210016
}

${ }^{*}$ Corresponding Author / E-mail: meeczhang@nuaa.edu.cn

\section{Abstract}

Elliptical vibration-assisted cutting (EVC) is a method for rapidly creating ultra-precision freeform surfaces with micro groove features, which have a wide array of industrial applications such as drag reduction, self-cleaning action, optical tuning behavior, etc. The complicated fabrication process and limited shape and deep control of micro-groove features by using EVC limit the applications of current EVC process in the fabrication of micro-groove. To overcome these limitations and improve overall performance, a layered elliptical vibration-assisted cutting (LEVC) process is presented to fabricate deep micro groove with different shape. First, a detailed layered elliptical vibration-assisted cutting method is described and a key step, the layered elliptical vibration-assisted tool path planning, is analyzed and established. Then the machining stress under the proposed LEVC method and layered conventional cutting (LCC) process were simulated and compared through finite element analysis. The simulation results show that the LEVC process can generate the lower residual stress compared to the LCC process. To validate the machining performance of the proposed LEVC process, the machining experiments for two groups of micro grooves under the proposed LEVC process and LCC process are performed. The performance tests and comparison results between the generated micro grooves under the proposed LEVC method and LCC process indicate that the proposed LEVC method is effective for generating micro groove with 
the controllable shape and depth. The actual experiments show that the machining capability of the developed LEVC method for micro groove is satisfactory.

Keywords: Micro groove, Elliptical vibration-assisted cutting, Layered tool path, Simulation, Machining stress

\section{Declarations}

Funding (information that explains whether and by whom the research was supported)

The work in this paper was supported by the National Natural Science Foundation of China

(NSFC) under Grant Number 51675277 and by Six Talent Peaks Project in Jiangsu Province under Grant Number GDZB-011.

Conflicts of interest/Competing interests (include appropriate disclosures)

The authors declare that they have no known competing financial interests or personal relationships that could have appeared to influence the work reported in this paper.

Availability of data and material (data transparency)

Not applicable

Code availability (software application or custom code)

Not applicable

Authors' contributions (optional: please review the submission guidelines from the journal whether statements are mandatory)

The contribution of this paper is that a layered elliptical vibration-assisted cutting ( LEVC) process is presented to fabricate deep micro groove with different shape on the complicated surface by using elliptical vibration-assisted cutting . 


\title{
Investigation on Layered Elliptical Vibration-Assisted Cutting of Micro Groove
}

\author{
Chen Zhang* \\ College of Mechanical and Electrical Engineering, Nanjing University of Aeronautics \\ and Astronautics, China, 210016
}

${ }^{*}$ Corresponding Author / E-mail: meeczhang@nuaa.edu.cn

\begin{abstract}
Elliptical vibration-assisted cutting (EVC) is a method for rapidly creating ultra-precision freeform surfaces with micro groove features, which have a wide array of industrial applications such as drag reduction, self-cleaning action, optical tuning behavior, etc. The complicated fabrication process and limited shape and deep control of micro-groove features by using EVC limit the applications of current EVC process in the fabrication of micro-groove. To overcome these limitations and improve overall performance, a layered elliptical vibration-assisted cutting (LEVC) process is presented to fabricate deep micro groove with different shape. First, a detailed layered elliptical vibration-assisted cutting method is described and a key step, the layered elliptical vibration-assisted tool path planning, is analyzed and established. Then the machining stress under the proposed LEVC method and layered conventional cutting (LCC) process were simulated and compared through finite element analysis. The simulation results show that the LEVC process can generate the lower residual stress compared to the LCC process. To validate the machining performance of the proposed LEVC process, the machining experiments for two groups of micro grooves under the proposed LEVC process and LCC process are performed. The performance tests and comparison results between the generated micro grooves under the proposed LEVC method and LCC process indicate that the proposed LEVC method is effective for generating micro groove with the controllable shape and depth. The actual experiments show that the machining capability of the developed LEVC method for micro groove is satisfactory.
\end{abstract}


Keywords: Micro groove, Elliptical vibration-assisted cutting, Layered tool path, Simulation, Machining stress

\section{Introduction}

The demand for creation of component surface with novel attributes that enhance product functional performance has recently been increasing in many industries. The surface's functions are controlled by the micro/nano-scale surface structures manufactured artificially for industrial uses such as in aerospace, illumination, tribology, biomedical, and other fields of engineering [1-4]. The fabrication processes for the micro/nano-scale surface structures include laser scribing [5], microelectrical discharge machining [6], micro-electrochemical machining [7], micro-end milling [8], micro-fly cutting [9], atomic-force-microscope scribing [10].

For generating geometrically defined surface textures, micromachining with diamond tools is a promising approach due to the merits of high form accuracy and flexibility [11-12]. With combining the elliptical vibration assisted cutting (EVC), micromachining provides a potential process for precision machining of complex micro-structured surfaces with wavelengths above tens of microns, which is difficult to achieve economically by other processes. Since the elliptical vibration assisted cutting (EVC) adds small-amplitude, high-frequency tool displacement to the cutting motion of tool, it brings a lot of machining advantages, such as suppression of burrs, reduction of tool wear, and enhanced material removal rate [13-14].

Suzuki et al. [15] proposed a new ultra-precision sculpturing method for micro/nano scale for difficult-to-cut materials by utilizing variations of an elliptical-vibration locus. Zhang et al. [16-17] further explored ultra-precision nano-structure fabrication with an amplitude-controlled sculpturing method in elliptical-vibration cutting considering a locus compensation. Ping et al. [18] focused on fast generation of dimples on cylindrical surfaces and obtained the micro-channeling topography with their texturing method. Gandhi et al. [19] investigated the ability to generate micro-scale textures with prescribed size and morphology by employing the control of underlying process parameters in vibration/modulation-assisted machining; novel surface textures including protrusions (ribs and fins) and depressions (dimples) were generated. Xu et al. [20] proposed a novel rotary 
ultrasonic texturing technique to fabricate hybrid micro/nano-textures on flat surfaces by using a tailored one-point diamond tool. With combination of fast-tool-servo and fly-cutting technology, the end-flying-cutting servo system is limited in its efficiency and adaptation of machining materials, for instance, it is not applicable to machine steel due to rapid tool wear and surface deterioration [21]. Kim and Loh [22] investigated the parameters of elliptical vibration cutting (EVC) in micro-V grooving considering variations of the elliptical cutting locus and the excitation frequency. Lee et al. [23] presented a simulation software for micro-pattern cutting. It models the micro-grooving in 3D, predicting the cutting force and optimizing the time for the roughing stage.

In spite of the considerable amount of work devoted to the fabrication of micro groove, there are some difficulties in their current fabrication process to obtain micro groove easily on wing surface with controllable shape and depth, which are required in actual industry application, e.g., rotary shafts for improving strength. Thus, for this type of application, the understanding of the generation mechanism of the elliptical vibration loci is a key point. Motivated by the above-mentioned problems, we target the exploration of new fabrication process of micro groove by using the ultrasonic elliptical vibration-assisted cutting with consideration of controllable shape and depth. Our previous studies have shown a novel fabrication method to obtain the dimple by using ultrasonic elliptical vibration cutting [24-25]. In this paper, a layered elliptical vibration assisted cutting method was proposed to fabricate micro-groove on the workpiece surface by only using the elliptical and feed motions of elliptical vibration tool and this process can be easily to obtain in current machining conditions with controllable shape and depth. A detailed layered elliptical vibrationassisted cutting method was described and the layered elliptical vibration-assisted tool path planning was analyzed and established. Then the machining stress under the proposed layered elliptical vibration-assisted cutting method and layered conventional cutting process was simulated and compared through finite element analysis. The simulation results showed that the layered elliptical vibration-assisted cutting process can generate the lower residual stress.

The remaining parts of the paper are organized as follows: Section 2 describes the design of the LEVC Method with its ideas of the LEVC method addressed in Section 2.1, The steps of the LEVC method in Section 2.2 and its The planning of the layered elliptical vibration-assisted tool path in 
Section 2.3. Section 3 is devoted to FEA locus simulation of the layered elliptical vibration-assisted tool path, while its performance evaluation and machining experiments about Fabrication of micro groove on wing surface are presented in Section 4. Conclusions are drawn in Section 5.

\section{Design of the LEVC Method}

\subsection{The basic ideas of the LEVC method}

In airplane, ship industry, wing surface is general used to obtain the minimum friction force. The fabrication of micro-groove on such wing surfaces can help to improve the drag reduction performance of airplane or ship. Elliptical vibration-assisted cutting (EVC) is a method for rapidly creating ultra-precision free-form surfaces with micro groove features in current conditions. The complicated fabrication process and limited shape control of micro-groove features by using EVC limit the applications of current EVC process in the fabrication of micro-groove. To overcome these limitations and improve overall performance, a layered elliptical vibration-assisted cutting process is presented to fabricate micro groove by considering the ultrasonic elliptical vibration assisted cutting process. The basic ideas of the proposed LEVC process is shown in Fig.1.

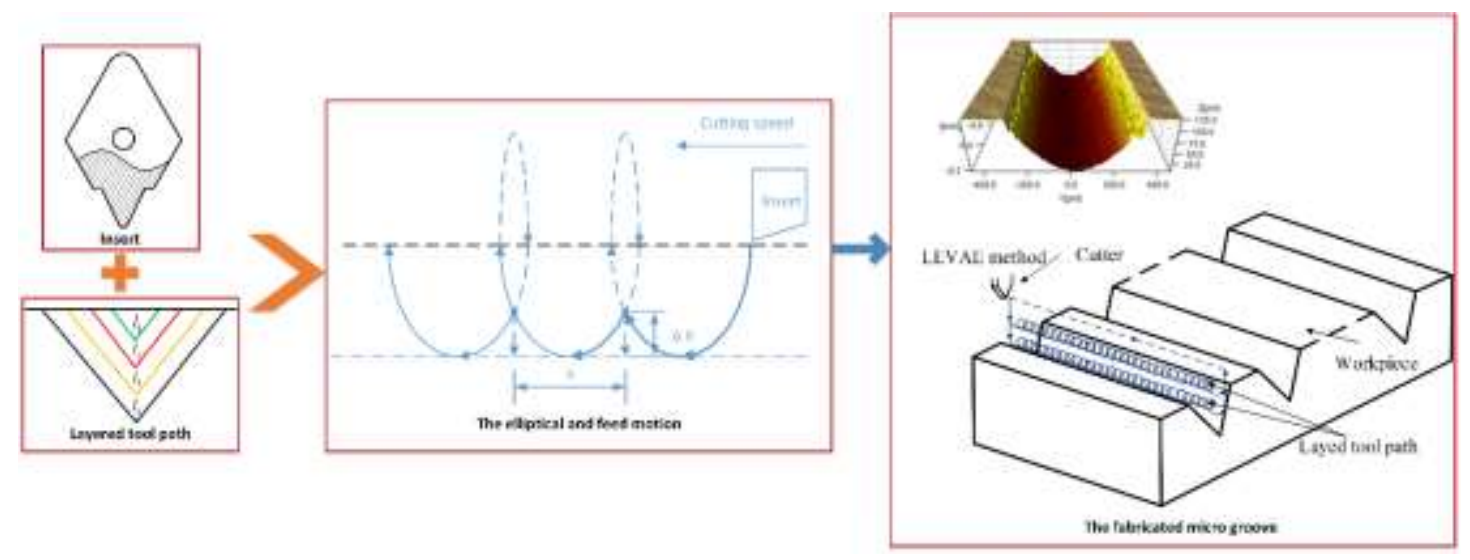

Fig.1 The schematic of the proposed layered elliptical vibration-assisted cutting process

In the proposed process, the fabrication motion of micro groove features on wing surface is generated by an elliptical tool, which is designed and manufactured by the authors. The elliptical and feed motions of elliptical vibration tool is simply used to fabricate the micro groove. In two motions, the elliptical motion is generated by the elliptical tool while the feed motion is obtained by 
using any machine tool in current industry conditions. Thus, the proposed process for fabricating micro groove is easily to realize in current machining conditions.

To obtain deep micro groove, a layered cutting method is utilized to fabricate micro groove on wing surface and micro groove with different depth can be obtained by choosing different layer number shown in Fig.1. The shape of micro groove can be generated by a modified insert and the modified insert with different shape can be used to obtain micro groove with different shape shown in Fig.1. Thus a controllable-shape deep micro groove can be fabricated based on the above ideas of the LEVC method.

\subsection{The steps of the layered EVC method}

According to the ideas of LEVC method, the steps of LEVC method can be described in details as follows:

Step 1: The micro-groove model for better drag reduction performance is designed by using FEM simulation and actual drag reduction experiments.

Step 2: A turning insert is selected and the shape of the turning insert is modified by using EDM process with consideration the designed micro-groove model and the modified turning insert is installed on elliptical vibration tool.

Step 3: The moving direction of the elliptical vibration tool is determined based on the feed direction of workpiece and the direction of elliptical vibration trajectory.

Step 4: The layered tool path planning of the designed elliptical vibration tool is generated with considering the moving direction of the elliptical vibration tool and micro-groove model.

Step 5: The topography model of micro-groove is established based on the generated ultrasonic elliptical vibration-assisted machining trajectory.

Step 6: The micro-groove topography is simulated to optimize the machining parameters and the actual machining experiments are conducted based on the optimized machining parameters.

In the above steps, the layered tool path planning is a key for generating the suitable microgroove in elliptical vibration-assisted cutting process. Thus, the following will discuss the layered elliptical vibration-assisted tool path planning. 


\subsection{The planning of the layered elliptical vibration-assisted tool path}

By analyzing the sharkskin surface structure, micro groove is selected as the optimal drag reduction structure. The micro groove model can be established as shown in Fig.2 by simplifying the sharkskin surface structure. The Fig.2 (a) is shown the simplified micro groove with width 1 , height $\mathrm{h}$ and apex angle $\theta$. In order to keep consistent pace with the actual machining, the section of bottom surface of micro groove is taken as a circular arc because of the tool nose radius $r$ such as shown in Fig.2 (b).

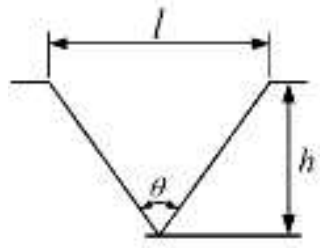

(a) Micro groove with tip angle

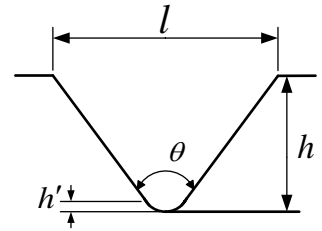

(b) Micro groove with circular arc

Fig.2. The section schematic of micro groove

Based on the tool nose radius $r$ and the apex angle $\theta$, the section height shown in Fig. 2(b) can be expressed as:

$$
h^{\prime}=r\left[1-\sin \left(\frac{\theta}{2}\right)\right]
$$

When the depth of micro V-groove $h$ is more than $h^{\prime}$, the width of micro V-groove 1 considering the symmetry of the micro groove shape can be calculated as:

$$
l=2\left(h-h^{\prime}\right) \tan \left(\frac{\theta}{2}\right)+2 r \cos \left(\frac{\theta}{2}\right)
$$

When the depth of micro V-groove $h$ is less than $h^{\prime}$, the width of micro groove $l^{\prime}$ can be written as :

$$
l^{\prime}=2 \sqrt{r^{2}-(r-h)^{2}}
$$

To realize the micro-groove cutting, the cutter should meet the following condition based on the cutting principle: 


$$
\left\{\begin{array}{c}
\alpha=\theta \\
c>\frac{h}{\cos \frac{\theta}{2}}
\end{array}\right.
$$

Where $\alpha$ is the tip angle of cutter and $c$ is the length of cutting edge.

If the used cutter can not satisfy the above conditions, the used cutter can be modified by using EDM until the modified cutter meets the above conditions.

Based on the established micro groove model, a uniform layered method of micro groove depth is designed to generate the cutting tool path in order to reduce the cutting force and improve the surface quality of micro groove. On each layer, the cutting depth is obtained by dividing the depth of micro groove uniformly.

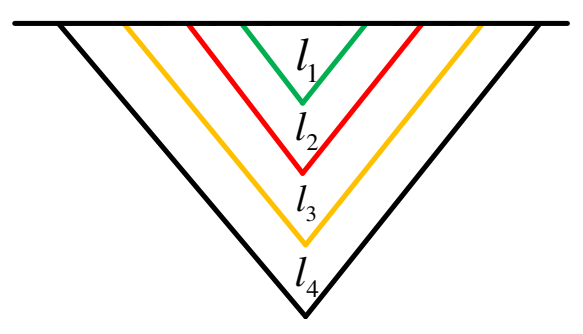

(a) Layered schematic of micro groove

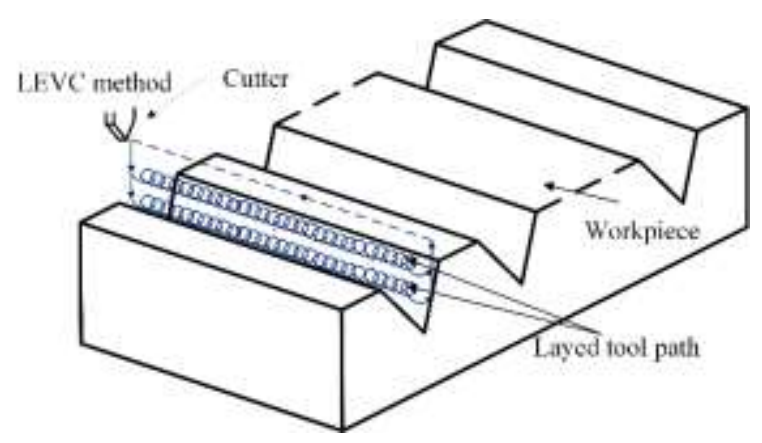

(b) The mode of LEVC

Fig.3 Layered elliptical vibration assisted cutting method

In order to obtain the micro groove, the maximum layered cutting depth is less than the tool nose radius $r$. Thus, the extrusion process can be obtained in the fabrication of micro groove to strengthen the surface of micro groove. Figure 3 (a) shows a layered schematic of micro groove. At the same time, the elliptical vibration-assisted cutting motion is added on the tool tip in the 
fabrication of micro groove to guarantee the suitable micro groove shape. Figure 3(b) shows the generation process of micro groove by adding elliptical vibration-assisted cutting. The left micro groove in Fig. 3 (b) shows the original shape without adding the elliptical vibration-assisted cutting motion on wing surface while the right micro groove in Fig. 3 (b) depicts the topography after the elliptical vibration-assisted cutting pass in the process. Compared to the left micro groove of Fig. 3(b), wavy micro groove is generated on the wing surface along the feed direction. As it can be seen, the topography of the micro groove on the surface in the feed direction is the result of the intersection of the neighboring elliptical loci. The above generation process can obtain a wide range of micro groove.

Based on the uniform layer, the numbers of layer $n$ can be obtained as:

$$
n=\frac{h}{d^{\prime}}
$$

Where $d^{\prime}$ is the cutting depth of each layer and is determined by the requirements of material machinability and machining quality of micro-groove.

\section{Locus simulation of the layered elliptical vibration- assisted tool path}

\subsection{Mesh generation}

To investigate the static and dynamic properties of the 3D EVC mechanism, i.e., its frequency response and static displacements, ANSYS was used to perform a FEM analysis. According to the established elliptical vibration-assisted tool path model in Fig. 3(a), the FE model is constructed as shown in Fig. 4. The $45^{\#}$ steel is assumed in modeling whose physical material parameters are: density $\left(7,800 \mathrm{~kg} / \mathrm{m}^{3}\right)$, Young's modulus (206 GPa) and Poisson's ratio (0.3).

Since the workpiece material is an elastic-plastic material, the strength and hardness of the tool are very large, and the strain is minimal during the entire machining process. Therefore, the tool is set to a rigid body in the simulation process. The constitutive model of the material adopts the most widely used Johnson-Cook model and the main considerations are the strain rate effect and 
temperature effect. It is more accurate to describe the deformation process of metal materials, and the form is simple and widely used. The specific equation is as follows:

$$
\bar{\sigma}=\left(A+B(\bar{\varepsilon})^{n}\right)\left[1+C \ln \left(\frac{\mathrm{d}}{\mathrm{E}}\right)\right]\left(1-\hat{\theta}_{0}^{m}\right)
$$

Where $\bar{\sigma}$ is the equivalent stress, $\bar{\varepsilon}$ is the equivalent plastic strain, $\dot{\bar{\varepsilon}}$ is the equivalent plastic strain rate, $\dot{\overline{\varepsilon_{0}}}$ is the reference strain rate, generally taken as $1 s^{-1}$, and $\mathrm{A}$ is the initial yield stress, $\mathrm{B}$ is the hardening modulus, $\mathrm{n}$ is the hardening index, $\mathrm{C}$ is the strain coefficient enhancement coefficient of the characterization material, and $\mathrm{m}$ is the thermal softening coefficient.

The formula for $\hat{\theta}$ is as follows:

$$
\hat{\theta}=\left\{\begin{array}{cc}
0 & \theta<\theta_{\text {transition }} \\
\frac{\left(\theta-\theta_{\text {transition }}\right)}{\left(\theta_{\text {melt }}-\theta_{\text {transition }}\right)} & \theta_{\text {transition }}<\theta<\theta_{\text {melt }} \\
1 & \theta>\theta_{\text {melt }}
\end{array}\right.
$$

Where $\theta$ is the current temperature, $\theta_{\text {transition }}$ is the transition temperature (the temperature at which the measurement experiment starts), and $\theta_{\text {melt }}$ is the material's melting point. Since the test usually starts at room temperature, the $\theta_{\text {transition }}$ is generally taken as $300 \mathrm{~K}$.

Table 1 the $45^{\#}$ steel's parameters of the Johnson-Cook model

\begin{tabular}{|l|l|l|l|l|l|l|l|}
\hline Material & $\mathrm{A}(\mathrm{MPa})$ & $\mathrm{B}(\mathrm{MPa})$ & $\mathrm{n}$ & $\mathrm{C}$ & $\mathrm{m}$ & $\theta_{\text {melt }}$ & $\theta_{\text {transition }}$ \\
\hline $45^{\#}$ steel & 507 & 320 & 0.064 & 0.28 & 1.06 & 1460 & 20 \\
\hline
\end{tabular}

In the three-dimensional orthogonal cutting model, the tool is set to a rigid body. The geometry of the workpiece is taken as $1 \mathrm{~mm} \times 0.8 \mathrm{~mm} \times 0.3 \mathrm{~mm}$, the tool's rake angle is $0^{\circ}$, the clearance angle is $7^{\circ}$, the tool nose radius is $0.04 \mathrm{~mm}$, and the tool thickness is $0.2 \mathrm{~mm}$. Tool material is carbide, the type of the mesh cell of tool is C3D8R, and a total of 34230 cells is obtained. The type of mesh cell of the workpiece is C3D8RT and a total of 21020 cells is obtained. The obtained FEA model is 
shown in Fig.4.

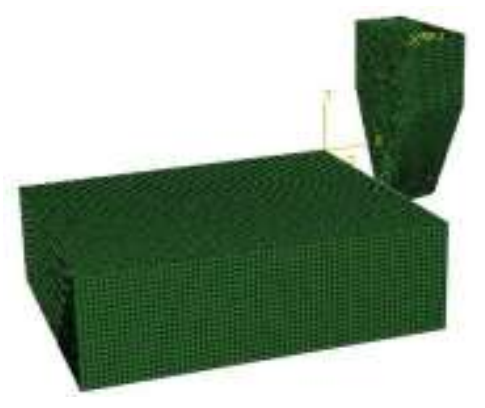

Fig.4 Finite element model of the layered elliptical vibration-assisted tool path

\subsection{Setup of boundary condition}

In the interaction, using the general algorithm that comes with ABAQUS, the contact type of the tool and the workpiece in the simulation process is set as the surface-surface contact; the mechanical constraint formulation is Penalty contact method, in the setting of the boundary condition. In order to prevent the workpiece from shifting, the bottom, the front and back surfaces of the workpiece must be fixed and restrained as shown in Fig. 5.

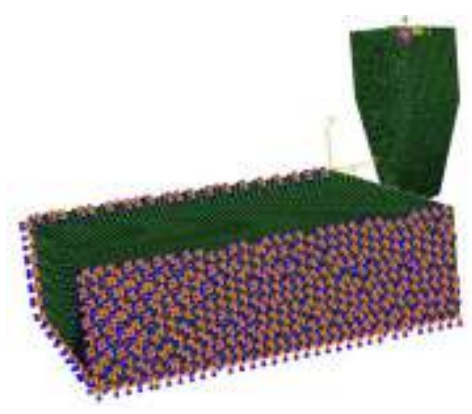

Fig.5 Finite element model with imposed boundary

In the boundary conditions, the setting of the layered trajectory is achieved by the setting of multiple analysis steps. The schematic diagram of the layered trajectory and analysis step setting is shown in Fig.6. To save computer resources, the number of layers in this simulation is 2 layers. In addition, in the analysis step of the tool cutting groove, the linear motion of the workpiece is applied to the tool. The Amplitude module in ABAQUS is utilized to add the elliptical vibration displacement of the tool in the xoy plane, and the Fourier series is used to represent each vibration 
of two directions in the Amplitude module. In both directions, the trajectory of the tool relative to the workpiece after adding elliptical vibration is shown in Fig.7. Two groups of simulation experiments with layered conventional cutting (LCC) and layered elliptical vibration-assisted cutting (LEVC) are conducted. The layered cutting parameters are shown in Table 2.

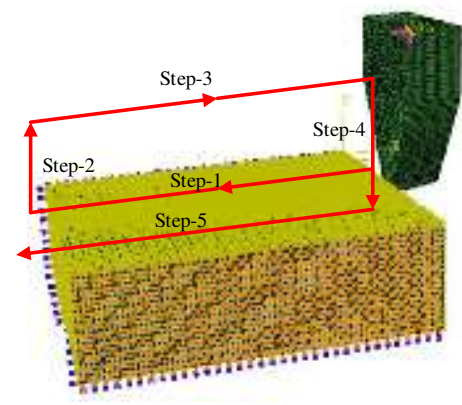

Fig.6 The multiple analysis steps in the cutting process
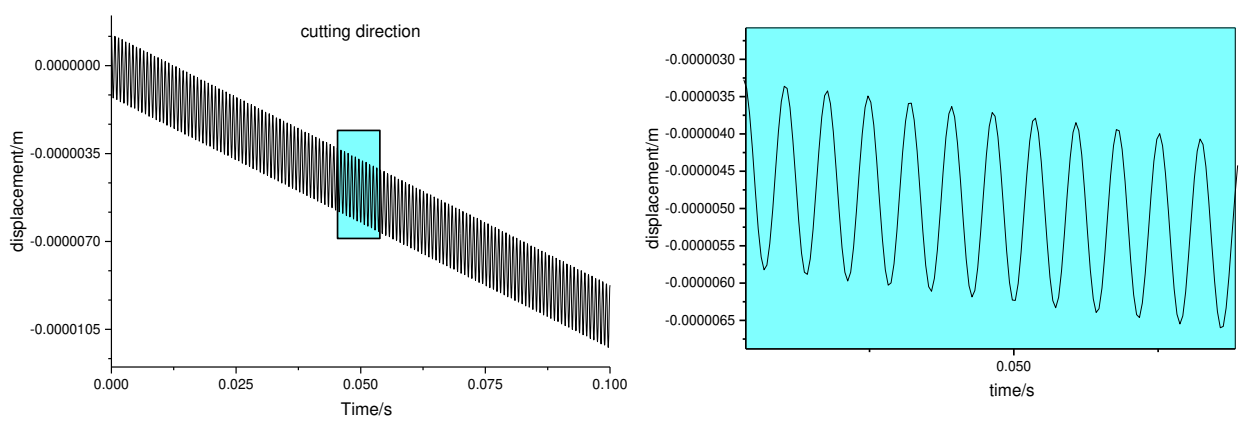

(a) $x$-direction (the cutting direction)
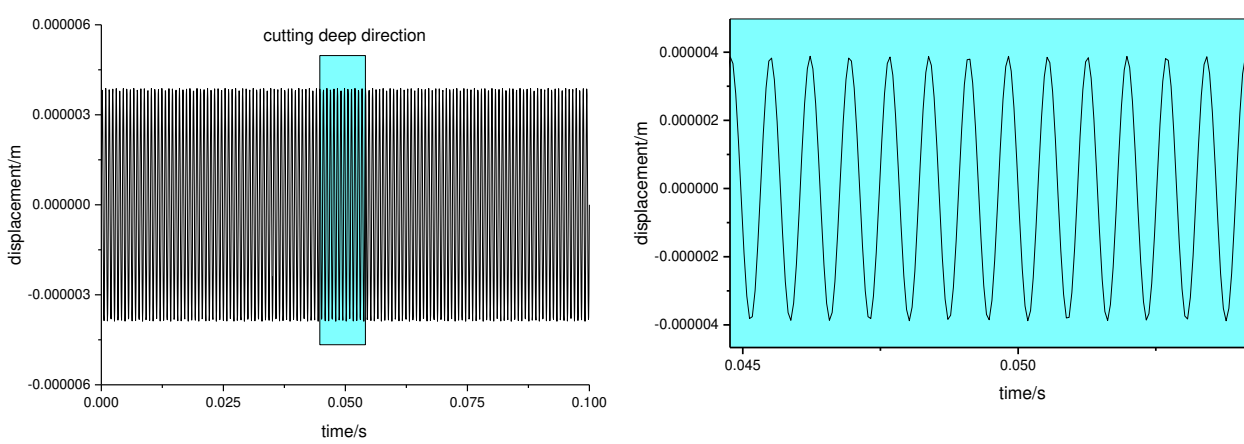

(b) $y$-direction (the cutting deep direction)

Fig .7 Tool trajectory of two-dimension with elliptical vibration assisted cutting 
Table 2 The parameters of the cutting simulation

\begin{tabular}{lcc}
\hline & LCC method & LEVC method \\
\hline Amplitude A(um) & 0 & 1.26 \\
\hline Amplitude B(um) & 0 & 3.88 \\
\hline Vibration frequency (kHz) & 0 & 18.6 \\
\hline Depth every layer(mm) & 0.02 & 0.02 \\
\hline Total cutting depth(mm) & 0.04 & 0.04 \\
\hline Number of layers & 2 & 2 \\
\hline Feed speed(mm/min) & 600 & 600 \\
\hline
\end{tabular}

\subsection{Analysis of simulation results}

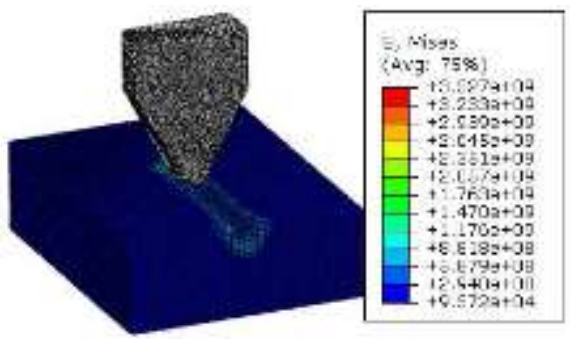

(a) Tool cutting at the first layer moment in LCC method

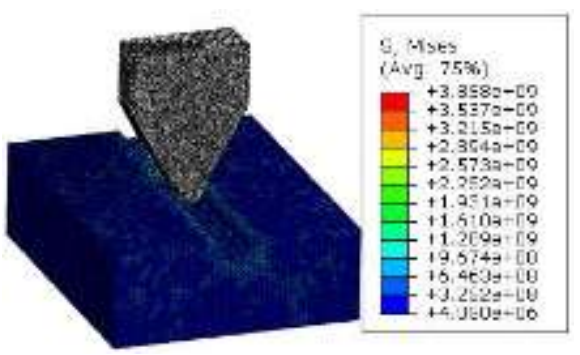

(b) Tool cutting at the second layer moment in LCC method 


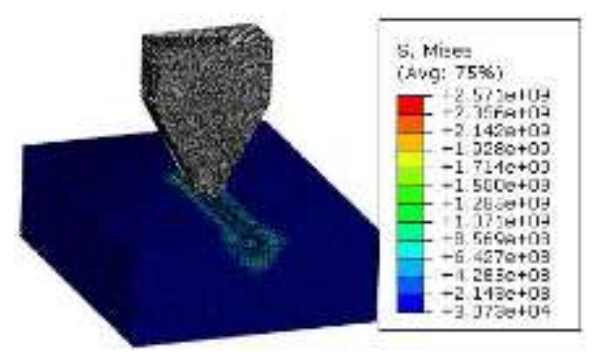

(c) Tool cutting at the first layer moment in LEVC method

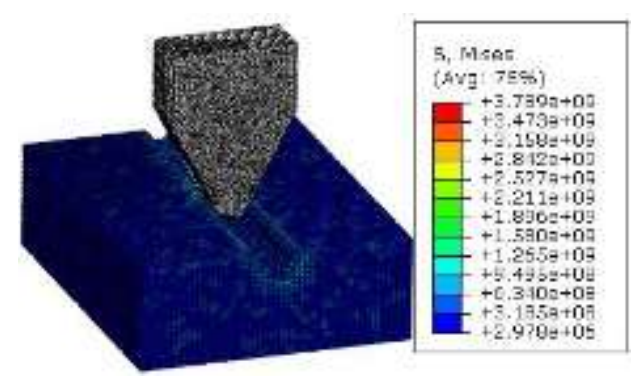

(d) Tool cutting at the second layer moment in LEVC method

Fig .8 Mises stress distribution under two cutting method

Figure 8(a) and Fig.8(b) show the Mises stress schematic at different times when cutting groove by using LCC method, and Fig.8(c) and Fig.8(d) give the Mises stress schematic through the LEVC method. It can be concluded from the Fig. 8 that the maximum Mises stress in cutting the second layer of the two cutting methods was larger than that in cutting the first layer, which was due to the presence of residual stress on the top layer after cutting. The Mises stress at the edge area of the groove is relatively concentrated from the Fig.8. Due to the presence of the stress, the plastic flow portion, which is not removed due to the cutting of the upper layer due to the presence of the stress, is easily detached to form defects or burrs, which affects the surface quality of the groove. In addition, the comparison shows that the maximum Mises stress is relatively stable during the LCC groove. The maximum stress of Mises varies greatly at different times when the LEVC method is adopted, and the average value is smaller than the value of the LCE method, it means that the residual stress of the groove surface of the LEVC is smaller, so the quality of the groove is better than that of LCC method. 


\section{Fabrication of micro groove on wing surface}

\subsection{Experimental setup}

Elliptical vibration-assisted micro groove machining experiments are carried out on FADAL VMC-8030HT vertical milling machine tool and the developed elliptical vibration apparatus is integrated into FADAL VMC-8030HT vertical milling machine tool (Fig. 9). The X-Y stage carries the workpiece while the Z-axis is aligned with the spindle axis. The motion generator of elliptical vibration is loaded in the spindle along the $\mathrm{Z}$-axis. The $\mathrm{X}$-Y stage provides the primary motion for the machining operation and controls the feed motion while the spindle sets the depth of cut. A dual channel function generator and amplifier generated the sinusoidal excitation voltages for the ultrasonic vibration generator.

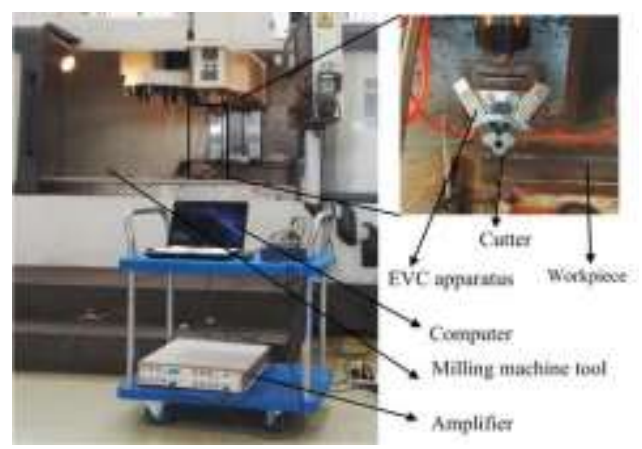

Fig.9 The schematic of UEVC machining system

Table 3 Experimental conditions

\begin{tabular}{ccc}
\hline \multirow{2}{*}{ Workpiece } & Material & No. 45 steel \\
\cline { 2 - 3 } & Shape & Wing surface \\
\cline { 2 - 3 } Tool & Material & Carbide \\
\cline { 2 - 3 } & Rake angle $\left({ }^{\circ}\right)$ & 0 \\
\cline { 2 - 3 } & Clearance angle $\left({ }^{\circ}\right)$ & 7 \\
\cline { 2 - 3 } Style & DCMT \\
\hline \multirow{2}{*}{ Vibrator } & Nose radius $(\mu \mathrm{m})$ & 18.1 \\
\cline { 2 - 3 } & Vibration frequency $(\mathrm{kHz})$ & 55 \\
\cline { 2 - 3 } & Phase difference $\left({ }^{\circ}\right)$ & \\
\hline
\end{tabular}

To investigate the elliptical vibration-assisted machining of micro groove, micro-texturing 
experiments using Stainless steel are performed by elliptical vibration cutting and extrusion process. The cutting tools used are commercial tungsten carbide inserts with a nose radius of $400 \mu \mathrm{m}$ with modification based on the shape of micro groove. The workpiece was a wing surface. The workpiece material used in the elliptical vibration-assisted extrusion machining is No. 45 steel. The resonant frequency and phase difference of elliptical vibrator are respectively $18.1 \mathrm{kHz}$ and $55^{\circ}$. The spindle speed is $0 \mathrm{rpm}$ in the machining experiments. Table 3 presents the experimental conditions used for elliptical vibration-assisted micro-texturing machining process.

\subsection{Generation of micro groove}

Three groups of micro groove experiments under different feed rates are designed to verify the proposed LEVC method on FADAL VMC-8030HT vertical milling machine tool. Table 4 shows the machining condition of three groups of micro V-groove with different feed rates for the LCC process and the LEVC process. The LCC process and the LEVC process are respectively conducted for 3 cased in Table 4. Figure 10(a) shows a typical machined workpiece with micro groove by using LEVC and Fig. 10(b) gives a 3D topography of micro groove by using LEVC method.

Under all experimental conditions listed above, the three groups of generated micro groove surface topographies are measured by using a 3D white light interferometer UP Dule. The actual machined surface topographies by using LCC and LEVC methods are respectively shown in Fig.11 through 15 with Table 4 parameter of No 1,2,3.

The simulation process of micro-textures only considers a perfect sharp of the tool nose edge and ideal geometry of tool edge and do not include tool wear, etc. Therefore, in the actual situation, there exists some differences of micro-texture size between the experiment results and simulation results.

Table 4 Machining parameters of micro groove under different feed rate.

\begin{tabular}{cccccccc}
\hline No & \multicolumn{1}{c}{1} & & 2 & & 3 \\
& & & & & & \\
\hline Machining mode & LCC & LEVC & LCC & LEVC & LCC & LEVC \\
\hline Feed velocity $(\mathrm{mm} / \mathrm{min})$ & 300 & 300 & 600 & 600 & 1000 & 1000 \\
\hline
\end{tabular}




\begin{tabular}{ccccccc}
\hline The depth of micro groove $(\mathrm{mm})$ & 0.1 & 0.1 & 0.1 & 0.1 & 0.1 & 0.1 \\
\hline Layer number & 5 & 5 & 5 & 5 & 5 & 5 \\
\hline The cutting depth of each layer $(\mathrm{mm})$ & 0.02 & 0.02 & 0.02 & 0.02 & 0.02 & 0.02 \\
\hline The length of micro groove $(\mathrm{mm})$ & 100 & 100 & 100 & 100 & 100 & 100 \\
\hline Vibration frequency $(\mathrm{kHz})$ & 0 & 18.6 & 0 & 18.6 & 0 & 18.6 \\
\hline
\end{tabular}

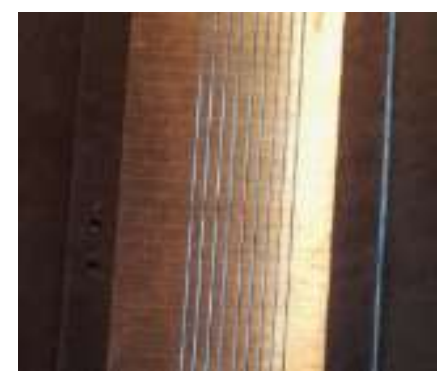

(a) The machined micro groove

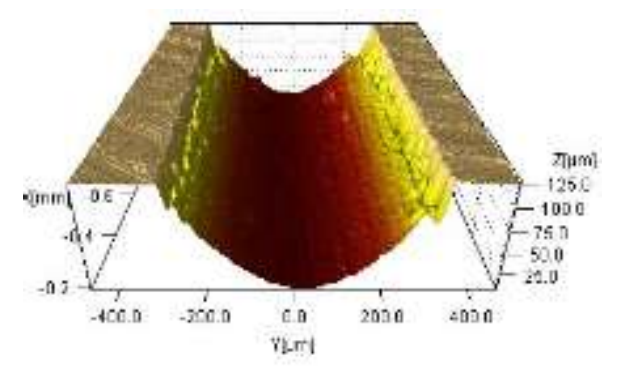

(b)The topography of micro groove

Fig. 10 The experimental results of the machined micro V-groove by using LEVC method

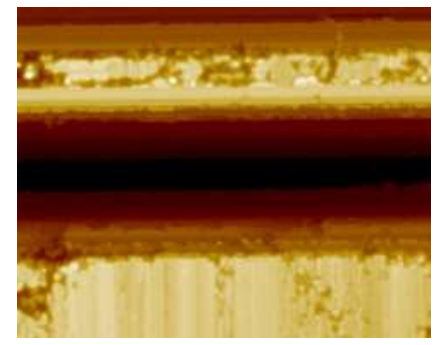

(a) LCC method

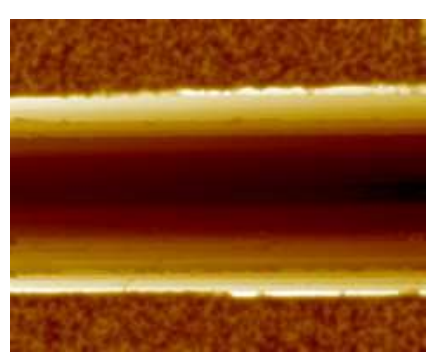

(b) LEVC method

Fig. 11 The comparison of micro groove surface topography with feed rate $300 \mathrm{~mm} / \mathrm{min}$.
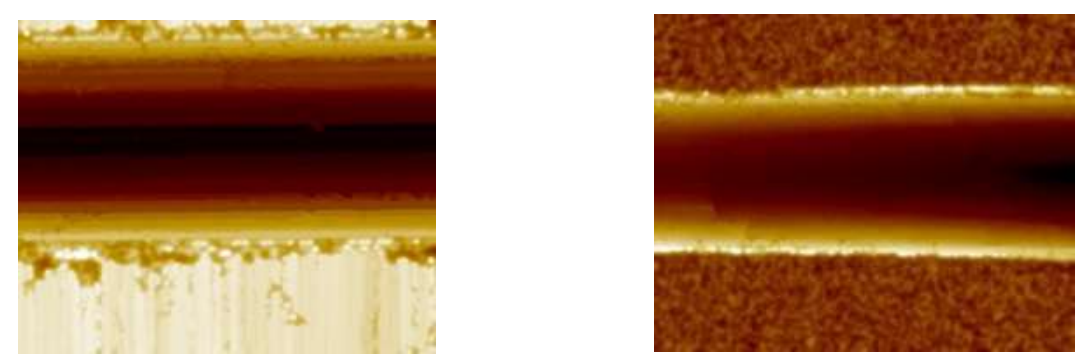
(a) LCC method

Fig. 12. The comparison of micro groove surface topography with feed rate $600 \mathrm{~mm} / \mathrm{min}$.

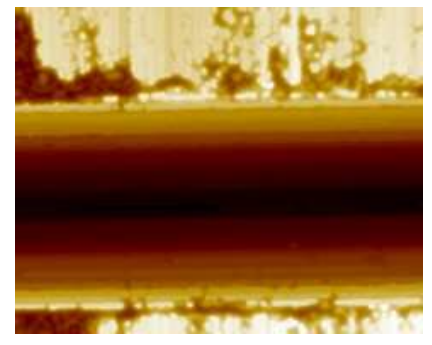

(a) LCC method

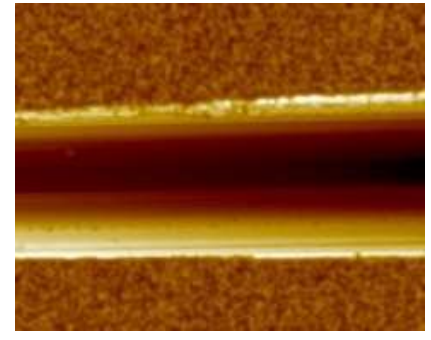

(b) LEVC method

Fig. 13The comparison of micro groove surface topography with feed rate $1000 \mathrm{~mm} / \mathrm{min}$.

\subsection{Error analysis of micro groove}

Figure 11 through Fig.13 have depicted three groups of micro groove topography corresponding to the three sets of experimental conditions listed in Table 4 for LCC and LEVC method. It can be observed from the image comparisons of the LCC and LEVC results in Fig.11, Fig.12 and Fig.13 that the results of the LCC and LEVC methods have similar waveforms and the section shapes while the topography of micro groove by LEVC method are smoother and are with less burr and defect, which means that the LEVC method has a higher accuracy for comparison of obtained micro groove.

In order to understand clearly the comparison results, a cross-section of machined micro groove by using LCC and LEVC method with feed rate $600 \mathrm{~mm} / \mathrm{min}$ is taken as example to verify the comparison effectiveness. Figure 14 gives the comparison of depth and width of micro groove between simulation results and actual experiment results of LCC and LEVC method. Besides, the error calculation formula is as follows:

$$
\delta=\frac{\left|l_{e x}-l_{\text {si m }}\right|}{l_{\text {si m }}} \times 100 \%
$$

The quantitative assessment of the simulated and generated micro-groove geometries for the conditions listed in Table 4 in terms of the depth of the micro groove, the width of the micro groove by using LCC and LEVC method based on the Eq. (4) is summarized in Table 5. 


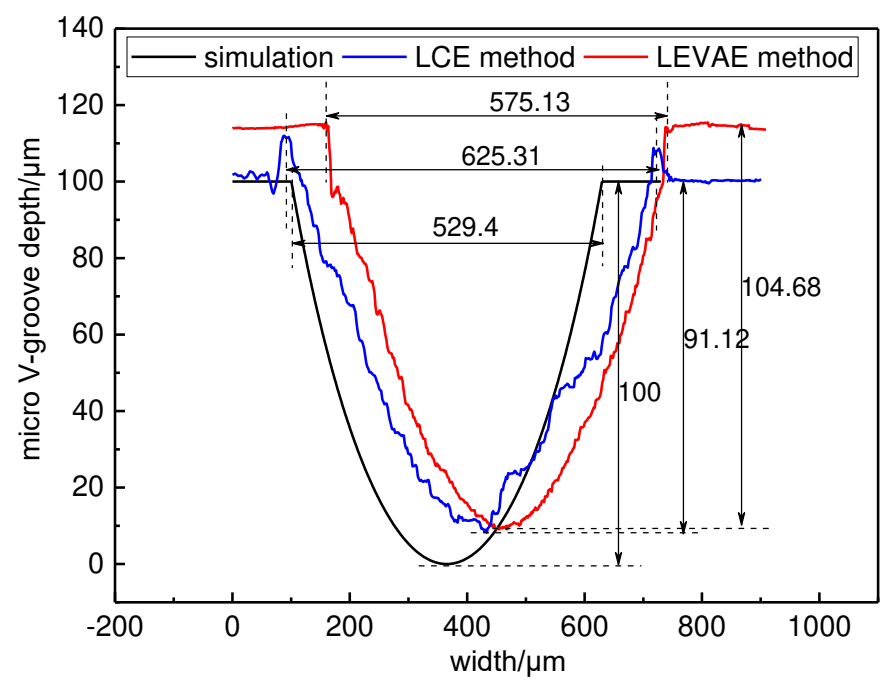

Fig.14 Error comparison of machined micro groove

Table 5 Error comparison of machined micro groove depth and width

\begin{tabular}{|c|c|c|c|c|c|c|}
\hline \multirow{2}{*}{ No } & \multicolumn{3}{|c|}{ Depth/$/ \mu \mathrm{m}$} & \multicolumn{3}{|c|}{ Width/ $\mu \mathrm{m}$} \\
\hline & Simulation & Experiment & Error & Simulation & Experiment & Error \\
\hline LCC & 100 & 91.12 & $8.88 \%$ & 529.4 & 625.31 & $18.12 \%$ \\
\hline LEVC & 100 & 104.68 & $4.68 \%$ & 529.4 & 575.13 & $8.64 \%$ \\
\hline
\end{tabular}

\subsection{Analysis and discussion}

It can be observed from the comparison of the simulation and experimental results in Table 5 and Fig. 14 that the relative error of the depth of the micro grooves from LCC method is within $8.88 \%$ and the actual depth of micro grooves from LCC method is less than that of simulation results while the relative error of the depth of the micro grooves from LEVC method is within $4.68 \%$ and the actual depth of micro grooves from LEVC method is more than that of simulation results. Similarly, for the width of the micro groove, the maximum error from LCC method is $18.12 \%$ and the actual width of micro grooves from LCC method is more than that of simulation results while the relative error of the width of the micro grooves from LEVC method is within $8.64 \%$ and the actual width of micro groove from LEVC method is more than that of simulation results. This can be explained that the established simulation model does not consider the actual physical factor influence on machining 
topography. The differences can be attributed to the influence of tool nose arc and the generated elliptical vibration in the cutting depth direction. Thus, the obtained depth of micro groove is more than the simulation result by LEVC method. Although the experimental results for the width of the micro grooves from LCC method and LEVC method qualitatively reflect the simulation results, the larger difference can be attributed to the way the measurements were taken. The measurements correspond to the projected image to a plane while the actual width of the micro groove in the longitudinal direction is along the wing's surface.

The error of the depth of micro groove is within $10 \%$ and the error of depth of micro groove by using LEVC method is less than that of LCC method. The same conclusion can be obtained from Table 5 and Fig. 14 that the error of width of micro groove by using LEVC method is less than that of LCC method. From all the above comparisons, it can be concluded that a close correspondence in the surface topography for micro groove exists between simulations and experiments in LEVC process.

The differences can be ascribed to the following aspects: (1) the actual material properties in the experiments and the material properties in simulation models are different; and (2) Machining precision of ultrasonic EVC apparatus will give rise to the error of experiment results; and (3) only the effect of geometric factors on micro-groove topography are included in the established simulation model omitting the influence of physical factors such as of the machining process on dimple topography.

The above comparisons show that the actual generated micro groove by the proposed LEVC method are consistent with the simulation results and verify that the proposed LEVC method is effective for generating deep micro groove. The resonant frequency, the amplitudes, and the resolution of the used ultrasonic EVC mechanism can be taken as reliable parameters for the prediction of the variant characteristics of the fabricated micro groove. The actual experiments show that the machining capability of the developed LEVC method for micro groove is satisfactory.

\section{Conclusions}

A novel LEVC method is proposed to obtain micro groove easily on wing surface with 
controllable shape and deep in this paper. The cutting performance of the proposed LEVC method for micro grooves is validated through simulation and cutting experiments. The summary of findings from the present work is as follows:

- A novel LEVC method by fabricating deep micro groove with the controllable shape is proposed by combining the ultrasonic elliptical vibration assisted cutting process and a detailed idea of the proposed LEVC method is described.

- The key step of the proposed LEVC method, the planning of the layered elliptical vibration-assisted tool path is analyzed and established by considering the fabrication of deep and controllable-shape micro grooves.

- The machining stress under the proposed LEVC method and LCC process were simulated and compared through finite element analysis. The simulation results show that the LEVC process can generate the lower residual stress compared to the LCC process.

- Two groups of micro-grooves cutting and simulation experiments were performed under the proposed LEVC process and LCC process. The performance tests and comparison results between the generated micro grooves under the proposed LEVC method and LCC process indicate that the proposed LEVC method is easy to generate the controllable-shape micro groove.

\section{Acknowledgments}

The work in this paper was supported by the National Natural Science Foundation of China (NSFC) under Grant Number 51675277 and by Six Talent Peaks Project in Jiangsu Province under Grant Number GDZB-011.

\section{References}

[1] A. Bruzzone, H. Costa, P. Lonardo, D. Lucca, Advances in engineered surfaces for functional performance, CIRP Annals - Manufacturing Technology, 2008, 57(2): 750-769. 
[2] F. Fang, X. Zhang, A. Weckenmann, G. Zhang, C. Evans, Manufacturing and measurement of freeform optics, CIRP Annals - Manufacturing Technology, 2013,62(2): 823-846.

[3] C. J. Evans, J. B. Bryan, 'Structured,' 'textured' or 'engineered' surfaces, CIRP Annals Manufacturing Technology,1999,48(2): 541-556.

[4] Z. C. Yang, L. D. Zhu, G. X. Zhang, C. B. Ni, B. Lin, Review of ultrasonic vibration-assisted machining in advanced materials, International Journal of Machine Tools \& Manufacture, 2020,156: 103594 .

[5] D. Dhupal, B. Doloi, B. Bhattacharyya, Modeling and optimization on Nd:YAG laser turned micro-grooving of cylindrical ceramic material. Optics and Lasers in Engineering 2009, 7:917925.

[6] H. S. Lim, Y. S. Wong, M. Rahman, M. K. Edwin Lee, A study on the machining of high-aspect ratio micro-structures using micro-EDM. Journal of Materials Processing Technology 2003,140:318-325.

[7] C. H. Jo, B. H. Kim, C. N. Chu, Micro electrochemical machining for complex internal micro features, CIRP Annals - Manufacturing Technology 2009, 58: 181-184.

[8] T. Schaller, L. Bohn, J. Mayer, K. Schubert, Microstructure grooves with a width of less than $50 \mathrm{~mm}$ cut with ground hard metal micro end mills. Precision Engineering, 1999, 23: 229-235.

[9] D. P. Adams, M. J. Vasile, A. S. M. Krishnan, Micro-grooving and micro-threading tools for fabricating curvilinear features. Precision Engineering, 2000, 24:347-356.

[10] K. A. Bourne, Development of a high-speed high-precision micro-groove cutting process, University of Illinois Urbana Champaign, 2011

[11] Zhu, W. L., Yang, S., Ju, B. F., Jiang, J. and Sun, A., "Scanning tunneling microscopy-based on-machine measurement for diamond fly cutting of micro-structured surfaces," Precision Engineering, vol. 43, pp. 308-314, 2016.

[12] Dornfeld, D., Min, S. and Takeuchi, Y., "Recent advances in mechanical micromachining," CIRP Annals- Manufacturing Technology, vol. 55, no. 2, pp. 745-768, 2006.

[13] Brehl, D.E and Dow, T.A, "Review of vibration-assisted machining," Precision Engineering, vol. 32, no. 3, pp. 153-172, 2008.

[14] Ma, C., Shamoto, E., Moriwaki, T., Zhang, Y. and Wang, L., "Suppression of burrs in turning with ultrasonic elliptical vibration cutting," International Journal of Machine Tools and Manufacture, vol. 45, no. 11, pp. 1295-1300,2005. 
[15] N. Suzuki, H. Yokoi, E. Shamoto, Micro/nano sculpturing of hardened steel by controlling vibration amplitude in elliptical vibration cutting. Precision Engineering, 2011, 35:44-50.

[16] J. G. Zhang, N. Suzuki, Y. Wang, E. Shamoto, Ultra-precision nano-structure fabrication by amplitude control sculpturing method in elliptical vibration cutting, Precision Engineering, 2015, 39: 86-99.

[17] J. G. Zhang, J. J. Zhang, A. Rosenkranz, N. Suzuki, E. Shamoto, "Frictional properties of surface textures fabricated on hardened steel by elliptical vibration diamond cutting," Precision Engineering, 2019,59: 66-72.

[18] P. Guo, Y. Lu, P. C. Pei, K. F. Ehmann, Fast generation of micro-channels on cylindrical surfaces by elliptical vibration texturing. Journal of Manufacturing Science and Engineering, 2014, 136(4):041008.

[19] R. Gandhi, D. Sebastian, S. Basu1, J. B. Mann, P. Iglesias, C. Saldana1, Surfaces by vibration/modulation-assisted texturing for tribological applications, The International Journal of Advanced Manufacturing Technology, 2016, 85(1) :1-12.

[20] S. Xu, K. Shimada, M. Mizutani, T. Kuriyagawa, Fabrication of hybrid micro/nano-textured surfaces using rotary ultrasonic machining with one-point diamond tool, International Journal of Machine Tools \& Manufacture, 2014, 86:12-17.

[21] S. To, Z. W. Zhu, W. H. Zeng, Novel end-fly-cutting-servo system for deterministic generation of hierarchical micro-nanostructures, CIRP Annals - Manufacturing Technology, 2015, 64 (1); 133-136.

[22] G. D. Kim, B. G. Loh, Characteristics of elliptical vibration cutting in micro-V grooving with variations in the elliptical cutting. Journal of Micromechanics and Microengineering, 2008, 18(2): 1-12.

[23] J. M. Lee, T. J. Je, D. S. Choi, S. W. Lee, D. Le, S. J. Kim, Micro grooving simulation and optimization in the roughing stage, International Journal of Precision Engineering and Manufacturing, 2010, 11(3):361-368

[24] C. Zhang, P. Guo, K. F. Ehmann, Y. Li, Effects of ultrasonic vibrations in micro-groove turning, Ultrasonics, 2016,67(4):30-40.

[25] C, Zhang, G, Shi, K. F. Ehmann ,Y. Li, Modeling and simulation of micro-groove topography on cylindrical surface by elliptical vibration-assisted turning, International Journal of Advanced Manufacturing Technology, 2016,86(5-8):1407-1427 